\title{
Pullbacks of hyperplane sections for Lagrangian fibrations are primitive
}

\author{
Ljudmila Kamenovat, Misha Verbitsky 2 \\ Dedicated to Professor Claire Voisin
}

\begin{abstract}
Let $p: M \rightarrow B$ be a Lagrangian fibration on a hyperkähler manifold of maximal holonomy (also known as IHS), and $H$ be the generator of the Picard group of $B$. Assume that $p$ has no multiple fibers in codimension 1 . We prove that $p^{*}(H)$ is a primitive class on $M$.
\end{abstract}

\section{Introduction}

In this paper we consider a compact hyperkähler manifold of maximal holonomy admitting a holomorphic fibration $\pi: M \rightarrow B$. The fibration structure is quite restricted due to the work of Matsushita, Mat, who first noticed that the general fiber is a Lagrangian abelian variety of half of the dimension of the total space. The base has the same rational cohomology as $\mathbb{C} P^{n}$ and the Picard group $\operatorname{Pic}(B)$ has rank one. Assume that multiple fibers of $\pi$ have codimension $\geqslant 2$ in $B$. We prove that the pullback of the fundamental class of a hyperplane section is primitive, i.e., indivisible as an integral class.

Theorem 1.1: Let $M$ be a hyperkähler manifold admitting a Lagrangian fibration $\pi: M \rightarrow B$ and $H$ be the generator of $\operatorname{Pic}(B)$. Assume that the set of points $b \in B$ with multiple fibers $\pi^{-1}(b)$ has codimension at least 2 . Then the class $\pi^{*} H \in H^{2}(M, \mathbb{Z})$ is primitive.

The proof is based on the observation that if $\pi^{*} H \in H^{2}(M, \mathbb{Z})$ is not primitive, i.e., $\pi^{*} H=m H^{\prime}$, then $H^{\prime}$ has trivial cohomology by Demailly, Peternell and Schneider's theorem. The assumption excluding multiple fibers is needed to exclude the case when $H^{\prime}$ is trivial on the generic fiber. Applying the Hirzebruch-Riemann-Roch formula for an irreducible hyperkähler manifold, one would obtain a contradiction.

\footnotetext{
${ }^{1}$ Partially supported by a grant from the Simons Foundation/SFARI (522730, LK).

${ }^{2}$ Partially supported by the Russian Academic Excellence Project '5-100'.
} 


\section{Basic notions}

Definition 2.1: A hyperkähler manifold of maximal holonomy (or irreducible holomorphic symplectic) manifold $M$ is a compact complex simply connected Kähler manifold with $H^{2,0}(M)=\mathbb{C} \sigma$, where $\sigma$ is everywhere non-degenerate.

For the rest of the paper we consider hyperkähler manifolds of maximal holonomy. Due to the work of Matsushita we know that the fibration structure of hyperkähler manifolds is quite restricted.

Theorem 2.2: (D. Matsushita, [Mat]) Let $M$ be a hyperkähler manifold and $f: M \rightarrow B$ a proper surjective morphism with a smooth base $B$. Assume that $f$ has connected fibers and $0<\operatorname{dim} B<\operatorname{dim} M$. Then $f$ is Lagrangian and $\operatorname{dim}_{\mathbb{C}} B=n$, where $\operatorname{dim}_{\mathbb{C}} M=2 n$.

Definition 2.3: Following Theorem 2.2, we call the morphism $f: M \rightarrow B$ a Lagrangian fibration on the hyperkähler manifold $M$.

Remark 2.4: In [Mat], D. Matsushita also proved that the base $B$ of a Lagrangian fibration has the same (rational) cohomology as $\mathbb{C} P^{n}$. In $[\mathrm{Hw}$, J.-M. Hwang proved that when $B$ is smooth, then it is actually isomorphic to $\mathbb{C} P^{n}$.

Definition 2.5: Given a hyperkähler manifold $M$, there is a non-degenerate primitive form $q$ on $H^{2}(M, \mathbb{Z}$ ), called the Beauville-Bogomolov-Fujiki form (or "BBF form" for short) of signature $\left(3, b_{2}-3\right)$, satisfying the Fujiki relation

$$
\int_{M} \alpha^{2 n}=c \cdot q(\alpha)^{n} \quad \text { for } \alpha \in H^{2}(M, \mathbb{Z})
$$

with $c>0$ a constant depending on the topological type of $M$. This form generalizes the intersection pairing on K3 surfaces. A detailed description of the form can be found in $[\mathrm{Be}], \mathrm{Bog}]$ and $[\mathrm{F}]$.

Definition 2.6: Let $f: M \longrightarrow B$ be a Lagrangian fibration. As shown in Mat,$H^{*}(B, \mathbb{Q})=H^{*}\left(\mathbb{C} P^{n}, \mathbb{Q}\right)$. Let $H$ be a primitive integer generator of $H^{2}(M, \mathbb{Q})$, and $\mathcal{O}(1)$ be a holomorphic line bundle on $B$ with first Chern class $H$. When $B=\mathbb{C} P^{n}$ (this is the case when $B$ is smooth by Hwang's 
result $[\mathrm{Hw}]$ ), the bundle $\mathcal{O}(1)$ coincides with the usual $\mathcal{O}(1)$. We call $H$ the fundamental class of a hyperplane section.

Remark 2.7: A semiample bundle is a base point free line bundle which has positive Kodaira dimension. Let $f: M \rightarrow B$ be a Lagrangian fibration, and $L:=f^{*}(\mathcal{O}(k)), k>0$. Clearly, $L$ is a semiample nef line bundle. By Matsushita's theorem any semiample nef line bundle is either ample or obtained this way. The $S Y Z$ conjecture (due to Tyurin, Bogomolov, Hassett, Tschinkel, Huybrechts and Sawon; see [V]) claims that the converse is also true: any nef line bundle on a hyperkähler manifold is either ample or semiample. This conjecture is a special case of Kawamata's abundance conjecture.

Remark 2.8: The Hirzebruch-Riemann-Roch formula for an irreducible hyperkähler manifold $M$ states that for a line bundle $L$ on $M, \chi(L)=$ $\sum a_{i} q\left(c_{1}(L)\right)^{i}$, where the coefficients $a_{i}$ are constants depending on the topology of $M$ (see [Hu, Section 1.11]). In particular, if $q\left(c_{1}(L)\right)=0$, then $\chi(L)=a_{0}=\chi\left(\mathcal{O}_{M}\right)=\sum(-1)^{i} h^{0, i}(M)=n+1$, where $2 n=\operatorname{dim} M$ (see [Hu, Section 1.7]).

Using the Hirzebruch-Riemann-Roch formula, we can easily obtain our main result for K3 surfaces. We are grateful to Claire Voisin for this observation.

Lemma 2.9: Let $S$ be a K3 surface with an elliptic fibration $\pi: S \rightarrow \mathbb{C} P^{1}$. Then the class $\pi^{*} \mathcal{O}(1) \in H^{2}(S, \mathbb{Z})$ is primitive.

Indeed, if we assume that $\pi^{*} \mathcal{O}(1)=m H$ for $m>1$, then $H$ would be $m$-torsion on all fibers and $h^{0}(H)=0$. By Serre duality, $h^{2}(H)=0$. Then applying the Hirzebruch-Riemann-Roch formula as in Remark 2.8, we obtain $2=\chi(H)=h^{0}(H)-h^{1}(H)+h^{2}(H)=-h^{1}(H) \leqslant 0$ - a contradiction.

Remark 2.10: This result was proven by E. Markman for manifolds of $\mathrm{K} 3^{[n]}{ }_{-}$ type ( $\mathrm{Mar}]$ ) and by B. Wieneck for generalized Kummer varieties (Lemma 2.7 in $[\mathrm{W}]$ ). We thank Klaus Hulek for pointing out this reference to us.

Here we restate a theorem by Demailly, Peternell and Schneider applied to compact Kähler manifolds with trivial canonical bundle, which is the set-up 
we need. The more general version of this result is Theorem 2.1.1 in [DPS]. This theorem was obtained under various hypotheses during the 1990s, see [Eno93] and [Mou99]. It was proved in [Tak97] when $L$ is nef.

Theorem 2.11: ([DPS, Corollary 2.1.2]) Let $(M, I, \omega)$ be a compact Kähler manifold, $K_{M}$ its canonical bundle, $\operatorname{dim}_{\mathbb{C}} M=N$, and $E$ a non-trivial nef line bundle on $M$. Assume that $E$ admits a Hermitian metric with semipositive curvature form. The cohomology class $\omega$ is considered as an element in $H^{1}\left(\Omega^{1} M\right)$. Consider the corresponding multiplication operator $\eta \longrightarrow \omega \wedge \eta$ maping $H^{p}\left(\Omega^{q} M \otimes E\right)$ to $H^{p+1}\left(\Omega^{q+1} M \otimes E\right)$. Then $\eta \longrightarrow \omega^{i} \wedge \eta$ induces a surjective map $H^{0}\left(\Omega^{N-i} M \otimes E\right) \rightarrow H^{i}\left(E \otimes K_{M}\right)$.

Further on, we shall also need the following trivial topological observation.

Claim 2.12: Let $M$ be a hyperkähler manifold of maximal holonomy. Then $H^{2}(M)$ is torsion-free.

Proof: The universal coefficients formula gives the exact sequence:

$$
0 \rightarrow \operatorname{Ext}_{\mathbb{Z}}^{1}\left(H_{1}(X ; \mathbb{Z}), \mathbb{Z}\right) \rightarrow H^{2}(X ; \mathbb{Z}) \rightarrow \operatorname{Hom}_{\mathbb{Z}}\left(H_{2}(X ; \mathbb{Z}), \mathbb{Z}\right) \rightarrow 0 .
$$

Since $H_{1}(X, \mathbb{Z})=0$ for a maximal holonomy hyperkähler manifold, this gives an isomorphism $H^{2}(X ; \mathbb{Z})=\operatorname{Hom}_{\mathbb{Z}}\left(H_{2}(X ; \mathbb{Z}), \mathbb{Z}\right)$, hence the torsion vanishes.

\section{Main Results}

An "abelian fibration" below is a holomorphic map with general fiber an abelian variety (or, in fact, a compact torus).

Proposition 3.1: Let $M$ be a smooth manifold admitting an abelian fibration $\pi: \quad M \rightarrow B$, and $E$ a line bundle on $M$ which is trivial on generic fibers and torsion on all fibers of $\pi$. Assume that multiple fibers of $\pi$ have codimension $\geqslant 2$ in $B$. Then $E=\pi^{*} E^{\prime}$, where $E^{\prime}$ is a line bundle on the base.

Proof: We need to show that each point $b \in B$ has a neighbourhood $U_{b}$ such that the restriction of $E$ to $\pi^{-1}\left(U_{b}\right)$ is a trivial bundle. 
In the sequel, we think of the restriction of $E$ to $\pi^{-1}\left(U_{b}\right)$ as of a holomorphic bundle underlying a local system. This is done by introducing an appropriate flat metric and proving that the monodromy of its Chern connection is trivial.

The bundle $E$ is torsion on all fibers of $\pi$, hence its tensor power $E^{\otimes k}$ is trivial on all fibers. Therefore, $E^{\otimes k}=\pi^{*} \pi_{*}\left(E^{\otimes k}\right)$ belongs to $\pi^{*}(\operatorname{Pic}(B))$. Given $b \in B$, choose a neighbourhood $U_{b} \subset B$ such that the restriction of $E^{\otimes k}$ to $\pi^{-1}\left(U_{b}\right)$ is trivial. To finish the proof it suffices to show that the restriction of $E$ to $W:=\pi^{-1}\left(U_{b}\right)$ is trivial.

Choose a constant metric $h^{k}$ on $\left.E^{\otimes k}\right|_{W}=\mathcal{O}_{W}$ and let $h$ be its $k$-th root, which is a metric on $\left.E\right|_{W}$. Since $h^{k}$ is constant, its curvature is flat, and the Chern connection $\nabla$ associated with $h$ is also flat.

To finish the proof, it remains to show that monodromy of $\nabla$ is trivial on all fibers. However, since $E$ is trivial on generic fibers, the monodromy of $E$ is trivial on generic fibers.

Any special fiber $F_{s}$ of $\pi$ is a deformation retract of its neighbourhood $U_{s}$ (see $[\mathrm{Mor}],[\mathrm{P},[\mathrm{C}]$ ). This retraction gives a map from the fundamental group of a general fiber to the fundamental group of the special fiber: the general fiber, denoted $F_{g}$, is embedded to $U_{s}$, which is then retracted to $F_{s}$. This map is clearly surjective if $F_{s}$ is a simple fiber. Therefore, the monodromy representation of $E$ on $F_{s}$ is induced by the monodromy of $E$ on $F_{g}$, which is trivial. We have shown that $\left.E\right|_{W}=\mathcal{O}_{W}$ in a neighbourhood of a simple fiber.

To deal with multiple fibers, we notice that they occur only in a subset $B_{0} \subset B$ of codimension $>1$. Outside of the set $B_{0}$ of multiple fibers, $E$ is the pullback of a bundle $E^{\prime}$ on $B \backslash B_{0}$. However, for any line bundle $L$, and for any subvariety $Z \subset M$ of codimension $\geqslant 2$, one has $L=j_{*} j^{*} L$, where $j: M \backslash Z \longrightarrow M$ is the open embedding (this is also called "Serre's condition S2", see OSS, Ch. II, Lemma 1.1.12]). With this observation, we see that $E$ is the pullback of a line bundle on the base $B$.

Theorem 3.2: Let $M$ be a hyperkähler manifold admitting a Lagrangian fibration $\pi: M \rightarrow B$, and $H$ the generator of $\operatorname{Pic}(B)$ (this group has rank 1, as shown by D. Matsushita; see Remark 2.4). Assume that multiple fibers of $\pi$ have codimension $\geqslant 2$ in $B$. Then the class $\pi^{*} H \in H^{2}(M, \mathbb{Z})$ is primitive.

Proof: Suppose that $\pi^{*} H$ is not primitive, and $\pi^{*} H=k H^{\prime}$ in $H^{2}(M, \mathbb{Z})$. 
Denote by $E$ the line bundle with $c_{1}(E)=H^{\prime}$. By Proposition 3.1, $E=\pi^{*} E^{\prime}$ unless $E$ is a non-trivial torsion bundle on general fibers of $\pi$. In the first case, $\pi^{*} H$ is primitive. This implies that $E$ is a non-trivial torsion bundle on smooth fibers of $\pi$, and torsion on all irreducible components of non-smooth fibers. In this case $c_{1}\left(E^{\prime}\right)=H$, which implies that $\pi^{*} H$ is primitive.

Let us apply the Enoki-Mourugane-Takegoshi-Demailly-Peternell-Schneider vanishing theorem (Theorem 2.11) to the manifold $M$ with the torsion nef line bundle $E$ to obtain the surjective map $H^{0}\left(\Omega^{2 n-i} M \otimes E\right) \rightarrow H^{i}(E)$. The bundle $T M$ restricted to a regular fiber $S$ of $\pi: M \longrightarrow B$ can be expressed as an extension

$$
\left.0 \longrightarrow T S \longrightarrow T M\right|_{S} \longrightarrow N_{S} \longrightarrow 0
$$

where $N_{S}$ is the normal bundle, which is trivial because $S$ is a fiber of the submersion $\pi: M \longrightarrow B$, in $s \in B$ which gives $N_{S}=\pi^{*} T_{s} B$. However, $T S$ is dual to $N_{S}$, because $S$ is Lagrangian, hence $\left.\Omega^{1} M\right|_{S}$ is an extension of trivial bundles. A tensor power of a trivial bundle is trivial, and therefore $\left.\Omega^{k} M\right|_{S}$ is also an extension of trivial bundles. Then $\left.\Omega^{k}(M)\right|_{S} \otimes E$ has no sections for all $k$, and Theorem 2.11 implies that $H^{i}(E)=0$ for all $i$.

To finish the proof, we apply the Hirzebruch-Riemann-Roch formula for the hyperkähler manifold $M$ with the line bundle $E$. Since $q(E)=q(L)=0$ and $H^{i}(E)=0$, from Remark 2.8 we obtain $n+1=\chi(E)=\sum(-1)^{i} \operatorname{dim} H^{i}(E)=$ 0 , a contradiction. Therefore, $\pi^{*} H$ is primitive.

\section{Applications}

In this section we describe some applications of the primitivity result.

Proposition 4.1: Let $M$ be a hyperkähler manifold admitting a Lagrangian fibration $f: M \rightarrow \mathbb{C} P^{n}$. Assume that multiple fibers of $f$ have codimension $\geqslant 2$ in $\mathbb{C} P^{n}$. Then the map $\pi_{2}(M) \longrightarrow \pi_{2}\left(\mathbb{C} P^{n}\right)$ is surjective.

Proof: Since we are in the settings of Theorem 3.2, we know that $L=$ $f^{*} \mathcal{O}(1)$ is primitive, i.e., $c_{1}(L)$ is not divisible. By Poincaré duality there is $\alpha \in H_{2}(M, \mathbb{Z})$ such that the pairing $\left\langle c_{1}(L), \alpha\right\rangle=1$ in $M$. This is the same as the pairing $\left\langle f_{*} \alpha, c_{1}(\mathcal{O}(1))\right\rangle$ in $\mathbb{C} P^{n}$, which means that $f_{*} \alpha$ is the class of a line, therefore $H_{2}(M, \mathbb{Z}) \longrightarrow H_{2}\left(\mathbb{C} P^{n}, \mathbb{Z}\right)$ is surjective. Since $M$ 
and $\mathbb{C} P^{n}$ are simply connected, this induces a surjection on the homotopy groups $\pi_{2}(M) \longrightarrow \pi_{2}\left(\mathbb{C} P^{n}\right)$ (see [Bre, Corollary 10.8]).

Remark 4.2: We conjecture that if the fibration $f: M \rightarrow \mathbb{C} P^{n}$ has no multiple fibers, then for a general curve $C \subset \mathbb{C} P^{n}$ there is a continuous section $C \rightarrow M$. The evidence is that for every curve class $[C]$ there is a class on $M$ surjecting to $[C]$ by Proposition 4.1.

Definition 4.3: A pullback of a very ample bundle is called very semiample.

Corollary 4.4: Let $E$ be a semiample line bundle on a hyperkähler manifold, which is not ample. Assume that the corresponding Lagrangian fibration has base $\mathbb{C} P^{n}$ and that the set of multiple fibers has codimension $\geqslant 2$ in $\mathbb{C} P^{n}$. Then $E$ is very semiample.

Proof: Indeed, by Theorem 3.2, $E=f^{*} \mathcal{O}(i)$, where $i>0$ and $f: M \longrightarrow \mathbb{C} P^{n}$ is a Lagrangian fibration.

Claim 4.5: Let $\pi_{1}, \pi_{2}$ be different Lagrangian fibrations on $M$ with base $\mathbb{C} P^{n}$, such that the sets of multiple fibers of both fibrations have codimension $\geqslant 2$ in $\mathbb{C} P^{n}$, and $P_{1}, P_{2}$ be non-trivial nef bundles which are trivial on the fibers of $\pi_{1}, \pi_{2}$. Then the natural map $F: M \longrightarrow \mathbb{P}\left(H^{0}\left(M, P_{1} \otimes P_{2}\right)^{*}\right)$ is holomorphic and birational to its image.

Proof: By Theorem 3.2, $P_{i}=\pi_{i}^{*} \mathcal{O}\left(k_{i}\right)$, for $i=1,2$. Since $P_{i}$ are nef and non-trivial, one has $k_{i}>0$, and the line bundle $P_{1} \otimes P_{2}$ is base point free. Therefore, the corresponding map $F: M \longrightarrow \mathbb{P}\left(H^{0}\left(M, P_{1} \otimes P_{2}\right)^{*}\right)$ is holomorphic. This map contracts only subvarieties $Z \subset M$ which lie in fibers of $\pi_{1}$ and $\pi_{2}$. Since the corresponding Lagrangian fibers are transversal when smooth, a general point is not contained in such a subvariety.

Acknowledgments. We are very grateful to Claire Voisin whose ideas inspired the proof of the main theorem. The work was completed at the SCGP during the second-named author's visit. We are grateful to the SCGP for the hospitality. The first named author thanks Michel Brion for their conversations about an earlier argument of the main theorem and for his interest. We would like to thank Christian Lehn for his interest and references. We are grateful to Ulrike Rieß for invaluable advice, her interest to 
this work, and for finding inconsistencies in an earlier version of our arguments. We thank the referee for their detailed comments and corrections. Much gratitude to D. Kaledin and D. Huybrechts for finding errors in the arxiv version and their suggestions.

\section{References}

[Be] Beauville, A., Varietes Kähleriennes dont la première classe de Chern est nulle. J. Diff. Geom. 18 (1983) 755 - 782.

[Bog] Bogomolov, F., Hamiltonian Kähler manifolds, Sov. Math. Dokl. 19 (1978) $1462-1465$.

[Bre] Bredon, G. E., Topology and Geometry, GTM 139, Springer (1997).

[C] Clemens, C. H. Degeneration of Kähler manifolds, Duke Math. J. 44 (1977), no. 2, 215-290.

[DPS] Demailly, J.-P., Peternell, T., Schneider, M., Pseudo-effective line bundles on compact Kähler manifolds, Int. J. Math. 12 (2001) 689 - 741.

[Eno93] I. Enoki, Strong-Lefschetz-type theorem for semi-positive line bundles over compact Kähler manifolds, Geometry and global analysis (Sendai, 1993), 211-212, Tohoku Univ., Sendai, 1993

[F] Fujiki, A., On the de Rham Cohomology Group of a Compact Kähler Symplectic Manifold, Adv. Stud. Pure Math. 10 (1987) 105 - 165.

[Hw] Hwang, J.-M., Base manifolds for fibrations of projective irreducible symplectic manifolds, Invent. Math. 174, No. 3 (2008) 625 - 644.

[HO] Hwang, Jun-Muk; Oguiso, Keiji, Characteristic foliation on the discriminant hypersurface of a holomorphic Lagrangian fibration, Amer. J. Math. 131 (2009), no. 4, 981-1007.

[Hu] Huybrechts, D., Compact hyper-Kähler manifolds: basic results, Invent. Math. 135 (1999) 63 - 113.

[Mar] Markman, Eyal, Lagrangian fibrations of holomorphic-symplectic varieties of $\mathrm{K}^{[n]}$-type, Algebraic and complex geometry, 241-283, Springer Proc. Math. Stat., 71, Springer, Cham, 2014. 
[Mat] Matsushita, D., On fibre space structures of a projective irreducible symplectic manifold, Topology 38 (1999), No. 1, 79 - 83. Addendum, Topology 40 (2001) No. 2, 431 - 432.

[Mat] Daisuke Matsushita Higher direct images of Lagrangian fibrations, arXiv:math/0010283, Amer J Math, 2005, 127: 243-259.

[M] Milne, J., Lectures on Etale cohomology, version 2.10. (2008).

[Mor] Morrison, David R. The Clemens-Schmid exact sequence and applications, Topics in transcendental algebraic geometry (Princeton, N.J., 1981/1982), 101-119, Ann. of Math. Stud., 106, Princeton Univ. Press, Princeton, NJ, 1984.

[Mou99] Ch. Mourougane, Théorèmes d'annulation génériques pour les fibrés vectoriels semi-négatifs, Bull. Soc. Math. Fr. 127 (1999) 115-133.

[OSS] Okonek, C., Schneider, M., Spindler, H., Vector bundles on complex projective spaces, Progress in mathematics, vol. 3, Birkhauser, 1980.

[P] Persson, Ulf, On degenerations of algebraic surfaces, Mem. Amer. Math. Soc. 11 (1977), no. 189.

[Tak97] K. Takegoshi, On cohomology groups of nef line bundles tensorized with multiplier ideal sheaves on compact Kähler manifolds, Osaka J. Math. 34 (1997) 783-802.

[V] Verbitsky, M., Hyperkahler SYZ conjecture and semipositive line bundles, arXiv:0811.0639, GAFA 19, No. 5 (2010) 1481-1493.

[W] Wieneck, B., Monodromy Invariants and Polarization Types of Generalized Kummer Fibrations, Math. Z. 290 (2018), no. 1-2, 347-378.

LuUdmila Kamenova

Department of Mathematics, 3-115

Stony Brook University

STONy Brook, NY 11794-3651, USA,

kamenova@math. sunysb.edu

Misha VERBitsky

Université libre de Bruxelles, CP 213,

Bd du Triomphe, 1050 Brussels, Belgium, also: 
Laboratory of Algebraic Geometry, National Research University HSE, Faculty of Mathematics, 7 Vavilova Str., Moscow, Russian Federation, verbit@verbit.ru 